\title{
Divergência genética entre acessos e cultivares de mamoneira por meio de estatística multivariada
}

\author{
Mauro Nóbrega da Costa ${ }^{(1)}$, Walter Esfrain Pereira(2), Riselane de Lucena Alcântara Bruno(1), \\ Eleusio Curvelo Freire ${ }^{(3)}$, Márcia Barreto de Medeiros Nóbrega ${ }^{(3)}$, Máira Milani $^{(3)}$ e Ademar Pereira de Oliveira ${ }^{(1)}$
}

(1)Universidade Federal da Paraíba (UFPB), Dep. de Fitotecnia, CEP 58397-000 Areia, PB. E-mail: costamn@terra.com.br, lane@cca.ufpb.br, ademar@cca.ufpb.br (2)UFPB, Dep. de Ciências Fundamentais e Sociais. E-mail: wep@cca.ufpb.br (3)Embrapa Algodão, Rua Osvaldo Cruz 1143, CEP 58107-720 Campina Grande, PB. E-mail: eleusio.fco@terra.com.br, nobrega@esalq.usp.br, maira@cnpa.embrapa.br

Resumo - O objetivo deste trabalho foi avaliar a divergência genética entre acessos e cultivares de mamoneira
(Ricinus communis L.) e utilizá-la como critério na escolha de genitores que viabilizem, a partir de hibridações, a
formação de populações segregantes. Os tratamentos foram representados pelos acessos BRA 4871, BRA 2968,
BRA 5550 e BRA 7722 Papo-de-gia, e as cultivares BRS 188 Paraguaçu, BRS 149 Nordestina, IAC-80, Mirante-10
e Pernambucana Melhorada. As características analisadas foram: início do florescimento (FR), número de racemos
por planta (NRP), comprimento efetivo do racemo primário (CR), altura de planta (AP), potencial produtivo (PP)
e teor de óleo nas sementes (TO). A divergência genética foi estimada por meio de estatística multivariada, com
base em variáveis canônicas e análise de agrupamento, tendo-se empregado a distância euclidiana média. Houve
a formação de dois grupos: o grupo I formado por oito genótipos e o grupo II por apenas um genótipo, a cultivar
Mirante-10. Apesar de a cultivar Mirante-10 ter sido a mais divergente, não deve ser recomendada para hibridação,
por sua baixa média de desempenho. As demais cultivares também apresentam restrições para hibridação, por
serem bastante similares. As variáveis que mais contribuíram para a divergência genética foram FR, AP, TO e CR. Termos para indexação: Ricinus communis, variação genética, agrupamento, análise multivariada.

\section{Genetic divergence on castor bean accesses and cultivars through multivariate analysis}

\begin{abstract}
This work aimed to evaluate genetic divergence among castor bean (Ricinus communis L.) cultivars, in order to enable the choice of parents which make the formation of segregating populations possible. Accesses BRA 4871, BRA 2968, BRA 5550 and BRA 7722 Papo-de-gia, and cultivars BRS 188 Paraguaçu, BRS 149 Nordestina, IAC-80, Mirante-10 and Pernambucana Melhorada were evaluated. Characteristics analyzed were: days to flowering, number of racemes per plant, length of pistillate region of main raceme, plant height, potential yield, and seed oil content. The genetic divergence among accesses and cultivars was studied by multivariate analysis techniques, with canonical variables and cluster analysis, making use of mean euclidean distance. Two groups were formed: group I, formed by eight genotypes; and group II, formed by one genotype, cultivar Mirante-10. In spite of being the more divergent, cultivar Mirante-10 should not be recommended for hybridization due to its low medium performance. The other cultivars also presented restrictions, as they were quite similar. The variables that more contributed to the genetic divergence were: days to flowering, length of pistillate region of main raceme, plant height, and seed oil content.
\end{abstract}

Index terms: Ricinus communis, genetic variation, cluster, multivariate analysis.

\section{Introdução}

A mamoneira (Ricinus communis L.) é uma oleaginosa de elevado valor socioeconômico e fonte de divisas para o país. Seus produtos e subprodutos são utilizados na indústria ou na agricultura, além de apresentar perspectivas de uso como fonte energética sob a forma de biodiesel.
Em determinadas áreas do Semi-Árido Nordestino, a mamoneira representa a cultura de sequeiro mais rentável, em razão das condições favoráveis ao seu desenvolvimento (Parente, 2003). Todavia, Moreira et al. (1996) ressaltaram que a maior dificuldade na exploração racional da mamona, no Nordeste do Brasil, está na baixa disponibilidade de sementes de cultivares adaptadas, 
produtivas, com elevado teor de óleo e tolerantes a pragas e doenças. Essas características identificadas e transferidas em programas de melhoramento que envolvam hibridações, resultam em práticas seletivas, ou ainda, na obtenção de híbridos $\mathrm{F}_{1}$ de expressivo vigor, ou heterose, decorrente do cruzamento entre dois genótipos de elevada capacidade combinatória e acentuada divergência genética entre si. A aplicação de técnicas eficientes para o reconhecimento e avaliação desses materiais faz-se necessária, uma vez que permite a obtenção de ganhos genéticos satisfatórios.

De acordo com Falconer (1981), a variabilidade genética de uma população segregante, necessária nos processos seletivos, é resultante da divergência genética entre os parentais envolvidos nos cruzamentos. Ainda, segundo esse autor, a expressão da heterose no híbrido intervarietal é dependente dos efeitos da dominância gênica, na manifestação do caráter e do quadrado da diferença da freqüência gênica nos genitores.

A hibridação representa uma técnica importante para o melhoramento de plantas, uma vez que possibilita a recombinação da variabilidade disponível, permitindo a obtenção de novos materiais, geneticamente superiores. A escolha dos parentais a serem utilizados em programas de hibridação e que possibilitem a formação de progênies superiores representa uma atividade indispensável que exige critérios e grande esforço do melhorista (Ramalho et al., 1993). No melhoramento da mamoneira, a hibridação tem sido muito utilizada e muitas cultivares já foram obtidas, com elevado valor das características de interesse comercial, por meio da seleção de segregantes genéticos (Savy Filho \& Banzatto, 1993; Freire et al., 2001; Savy Filho, 2005).

Quanto ao desenvolvimento de sementes híbridas de mamona, no Brasil, ainda há pouco interesse, em decorrência do baixo nível tecnológico utilizado no cultivo da mamoneira por produtores dessa oleaginosa (Savy Filho, 1999). Atualmente, nos Estados de Mato Grosso e Goiás, quatro híbridos comerciais vêm sendo cultivados como cultura de "safrinha", em sucessão à soja ou ao milho (Savy Filho, 2005). Em países de agricultura desenvolvida, como os Estados Unidos, a produção de híbridos comerciais é muito utilizada (Moreira et al., 1996; Freire et al., 2001).

Os estudos a respeito de divergência genética fornecem parâmetros para a identificação de genitores favoráveis à obtenção de populações segregantes, em programas de hibridação, que favorecem a seleção de genótipos superiores e, como conseqüência, a obtenção de populações geneticamente melhoradas. A divergência genética, avaliada por meio de processos preditivos ou técnicas multivariadas, tem merecido destaque, uma vez que dispensam a obtenção de híbridos, como nas análises dialélicas, que requerem um grande número de cruzamentos (Miranda et al., 2003; Cruz et al., 2004).

A avaliação da divergência genética, como critério para a escolha de genitores em programas de melhoramento, foi relatada por diversos autores a exemplo de Maluf et al. (1983), Miranda et al. (1988), Dias \& Kageyama (1997), Vidigal et al. (1997), Ribeiro et al. (1999) e Ferrão et al. (2002). Em relação à mamona, poucos estudos têm sido desenvolvidos quanto à divergência genética. Como exemplo tem-se o trabalho de Figueiredo Neto et al. (2004), com ênfase no manejo e uso adequado de germoplasma.

As técnicas de análise de agrupamento têm por objetivo dividir, seguindo-se algum critério de similaridade ou dissimilaridade, um grupo original de observações em vários outros grupos (Cruz et al., 2004); tais técnicas podem ser complementadas com a análise de variáveis canônicas. Esta última análise objetiva a simplificação estrutural dos dados amostrais, de forma que diferenças entre tratamentos, em princípio influenciadas por um conjunto maior de variáveis, possam ser avaliadas em espaços bidimensionais ou tridimensionais de fácil interpretação geométrica, o que possibilita a identificação de grupos similares em estudos de divergência genética (Cruz, 1990; Cruz et al., 2004). Conforme esses autores, as medidas de distâncias mais utilizadas na identificação de genitores para a hibridação são as distâncias euclidianas e a de Mahalanobis; as primeiras são recomendadas nos casos em que as unidades para o cálculo sejam escores de componentes principais ou de variáveis canônicas, e a distância euclidiana média é preferida em razão do número de variáveis.

O objetivo deste trabalho foi avaliar a divergência genética entre acessos e cultivares de mamoneira, com uso da análise multivariada, a fim de possibilitar a escolha de genitores que, a partir de hibridações, viabilizem a formação de populações segregantes para a seleção de genótipos favoráveis à obtenção de novas cultivares.

\section{Material e Métodos}

Este trabalho foi conduzido no período compreendido entre os meses de maio e novembro de 2002, na Fazenda Chã-de-Jardim, pertencente à Universidade Federal da Paraíba, no Município de Areia, PB, a 658'12"S e 
3542'15"W e 618 m de altitude, com precipitação média anual de $1.400 \mathrm{~mm}$ e temperatura entre 23 e $24^{\circ} \mathrm{C}$, com variações mensais mínimas.

O solo da área experimental é Argissolo VermelhoAmarelo eutrófico. Foram utilizados no plantio: uréia, $20 \mathrm{~kg} \mathrm{ha}^{-1} ; \mathrm{P}_{2} \mathrm{O}_{5}, 40 \mathrm{~kg} \mathrm{ha}^{-1} ; \mathrm{K}_{2} \mathrm{O}, 20 \mathrm{~kg} \mathrm{ha}^{-1}$; e, em cobertura, $40 \mathrm{~kg} \mathrm{ha}^{-1}$ de uréia. Foram utilizados $44 \mathrm{~kg}$ de esterco bovino por parcela, distribuídos em $4 \mathrm{~kg}$ por cova de $400 \mathrm{~cm}^{2}$ por $20 \mathrm{~cm}$ de profundidade.

O delineamento utilizado foi o de blocos ao acaso, com quatro repetições e nove tratamentos constituídos pelos acessos BRA 4871, BRA 2968, BRA 5550 e BRA 7722 Papo-de-gia e as cultivares BRS 188 Paraguaçu, BRS 149 Nordestina, IAC-80, Mirante-10 e Pernambucana Melhorada, procedentes do banco ativo de germoplasma da Embrapa Algodão, distribuídos em parcelas de $10 \mathrm{~m}$ de comprimento, em espaçamento de $2 \mathrm{~m}$ entre fileiras e $1 \mathrm{~m}$ entre covas.

Aos 25 dias após a semeadura, foi realizado o desbaste, tendo deixado uma planta por cova. A parcela ficou, então, constituída por 11 plantas, das quais foram avaliadas as seguintes características: início do florescimento (FR), número de racemos por planta (NRP), comprimento efetivo do racemo primário (CR), altura da planta (AP), potencial produtivo (PP) e teor de óleo nas sementes (TO), conforme Veiga et al. (1989) e Nóbrega et al. (2001). A determinação do teor de óleo nas sementes foi realizada por meio da técnica de espectroscopia por ressonância magnética nuclear (RMN) (Paz, 1996).

Todos os racemos foram protegidos com sacola de plástico telado, no início da maturação dos frutos, com o intuito de reduzir perdas pela deiscência de sementes em campo, eliminar a prática de complementação de secagem em terreiro ou secadores apropriados, bem como reduzir o número de colheitas.
Os dados foram, inicialmente, submetidos à análise de variância univariada, para avaliação da existência de variabilidade genética entre os genótipos e, em seguida, à análise de variância multivariada para a interpretação e avaliação da variabilidade genética global existente entre os mesmos genótipos. Essas análises foram processadas mediante o programa computacional SAEG (Ribeiro Junior, 2001), e as médias entre os tratamentos foram comparadas pelo teste de agrupamento de Scott \& Knott (1974) a 5\% de probabilidade.

As variáveis canônicas foram obtidas a partir da análise de variância multivariada, com matrizes de covariâncias fenotípicas e de covariâncias residuais entre tratamentos; a divergência genética, entre tratamentos, foi determinada com base na análise de agrupamento e de variáveis canônicas apresentadas por Cruz et al. (2004). A distância euclidiana média foi utilizada como medida de dissimilaridade. Aplicou-se na delimitação dos grupos a técnica de otimização de Tocher, cujo procedimento mantém a distância média intragrupo inferior à qualquer distância média intergrupo. Em relação à análise de variáveis canônicas, a divergência genética foi evidenciada pela dispersão dos escores, em gráfico com eixos representados pelas duas primeiras variáveis canônicas.

\section{Resultados e Discussão}

Pela análise de variância univariada, houve diferenças significativas entre tratamentos para quase todos os caracteres, exceto para número de racemos por planta, o que indica, a princípio, que há divergência entre acessos e cultivares. As médias apresentadas na Tabela 1 não mostraram diferenças significativas, pelo teste de Scott \& Knott (1974), para número de racemos por planta, o

Tabela 1. Médias dos quatro acessos e cinco cultivares, relativas a seis caracteres em mamona ${ }^{(1)}$.

\begin{tabular}{|c|c|c|c|c|c|c|}
\hline Acessos e cultivares & $\begin{array}{c}\text { FR } \\
\text { (dias) }\end{array}$ & NRP & $\begin{array}{l}\text { CR } \\
(\mathrm{cm})\end{array}$ & $\begin{array}{l}\text { AP } \\
(\mathrm{cm})\end{array}$ & $\begin{array}{c}\text { PP } \\
\left(\mathrm{kg} \mathrm{ha}^{-1)}\right)\end{array}$ & $\begin{array}{l}\text { TO } \\
(\%)\end{array}$ \\
\hline BRA 4871 & $57,5 \mathrm{~d}$ & $10,1 \mathrm{a}$ & $7,7 \mathrm{c}$ & $280,5 a$ & $1.709,7 \mathrm{a}$ & $50,1 \mathrm{~b}$ \\
\hline BRA 2968 & $55,3 d$ & $10,8 \mathrm{a}$ & $6,1 \mathrm{c}$ & $234,8 b$ & $1.882,6 \mathrm{a}$ & $51,6 \mathrm{a}$ \\
\hline BRA 5550 & $80,0 \mathrm{a}$ & $7,6 \mathrm{a}$ & $6,7 \mathrm{c}$ & $248,3 b$ & $1.159,7 b$ & $50,4 b$ \\
\hline BRA 7722 Papo-de-gia & $73,8 b$ & $6,9 a$ & $7,7 \mathrm{c}$ & $290,8 \mathrm{a}$ & $1.068,3 \mathrm{~b}$ & $48,8 \mathrm{c}$ \\
\hline BRS 188 Paraguaçu & $57,8 \mathrm{~d}$ & $9,9 a$ & $12,6 \mathrm{a}$ & $232,5 b$ & $2.181,1 \mathrm{a}$ & $52,6 \mathrm{a}$ \\
\hline BRS 149 Nordestina & $69,3 c$ & $8,6 \mathrm{a}$ & $7,3 \mathrm{c}$ & $250,3 b$ & $1.748,4 \mathrm{a}$ & $50,3 \mathrm{~b}$ \\
\hline IAC-80 & $53,3 \mathrm{~d}$ & $12,1 \mathrm{a}$ & $10,1 b$ & $270,0 \mathrm{a}$ & $2.299,3 \mathrm{a}$ & $51,8 \mathrm{a}$ \\
\hline Mirante-10 & $39,0 \mathrm{e}$ & $8,4 \mathrm{a}$ & $8,0 \mathrm{c}$ & $227,8 b$ & $800,2 b$ & $47,1 d$ \\
\hline Pernambucana Melhorada & $56,3 \mathrm{~d}$ & $11,8 \mathrm{a}$ & $6,2 \mathrm{c}$ & $234,8 b$ & $2.034,5 \mathrm{a}$ & $51,1 \mathrm{~b}$ \\
\hline
\end{tabular}

(1)FR1: início do florescimento; NRP: número de racemos por planta; CR: comprimento efetivo do racemo primário; AP: altura da planta; PP: potencial produtivo; TO: teor de óleo das sementes; médias com letras iguais não diferem entre si, pelo teste de Scott \& Knott, a 5\% de probabilidade. 
que confirma os resultados obtidos pelo teste $\mathrm{F}$ para este caráter. A característica início do florescimento (FR) apresentou maior variabilidade, seguida de teor de óleo das sementes (TO) e comprimento efetivo do racemo primário (CR). A altura de planta (AP) e o potencial produtivo (PP) foram representados por dois grupos, e o número de racemos por planta (NRP) por apenas um grupo. Isto evidencia pouca variabilidade no material em estudo, fator que diminui as chances de seleção. As cultivares BRS 188 Paraguaçu e IAC-80 e o acesso BRA 2968 apresentaram melhor desempenho médio para precocidade, maior produtividade e elevado teor de óleo.

As duas primeiras variáveis canônicas explicaram 94,39\% da variação total (Tabela 2), o que demonstra valor satisfatório para sua utilização nas análises posteriores (Cruz et al., 2004).

A primeira variável canônica encontra-se positivamente relacionada ao início do florescimento, altura da planta e teor de óleo na semente, enquanto a segunda representa um contraste entre o comprimento efetivo do racemo primário e teor de óleo com a altura da planta, uma vez que essas variáveis apresentam os maiores coeficientes de ponderação, com sinais contrários.

A amplitude das distâncias euclidianas médias apresentou valor máximo de 14,64 entre a cultivar
Mirante-10 e o acesso BRA 5550, e valor mínimo de 0,42 entre Pernambucana Melhorada e BRA 2968 (Tabela 3). Em termos genéticos, conclui-se que a cultivar Mirante-10 e o acesso BRA 5550 são os mais divergentes, ao passo que Pernambucana Melhorada e BRA 2968 são os mais similares.

No agrupamento pelo método de Tocher verificou-se a formação de apenas dois grupos, os quais são apresentados na Tabela 4. O grupo I foi representado pelos acessos BRA 4871, BRA 2968, BRA 5550 e BRA 7722 Papo-de-gia e as cultivares BRS 188 Paraguaçu, BRS 149 Nordestina, IAC-80 e Pernambucana Melhorada, e o grupo II foi representado pela cultivar Mirante-10. A distância intergrupos foi de 10,72 e a intragrupo foi de 5,88 para o grupo I.

Resultados satisfatórios seriam esperados nos cruzamentos entre a cultivar Mirante-10 e os demais genótipos e, especialmente, entre esta e o acesso BRA 5550. Todavia, verificou-se baixo desempenho médio da cultivar Mirante-10, quanto ao potencial produtivo e teor de óleo na semente, com superioridade apresentada apenas para precocidade (Tabela 1). Apesar de constituírem o mesmo grupo, consideráveis distâncias foram observadas entre BRA 5550 e BRA 4871, BRA 2968, BRS 188 Paraguaçu, IAC-80 e Pernambucana Melhorada, bem como entre BRA 7722

Tabela 2. Variáveis canônicas, obtidas a partir da análise de seis caracteres em mamona(1).

\begin{tabular}{|c|c|c|c|c|c|c|c|c|}
\hline \multirow{2}{*}{$\begin{array}{l}\text { Variável } \\
\text { canônica }\end{array}$} & \multirow{2}{*}{$\begin{array}{l}\text { Variância } \\
\text { (autovalor) }\end{array}$} & \multirow{2}{*}{$\begin{array}{c}\text { Variância } \\
\text { acumulada (\%) }\end{array}$} & \multicolumn{6}{|c|}{ Coeficiente de ponderação associado a: } \\
\hline & & & FR & NRP & $\mathrm{CR}$ & AP & PP & TO \\
\hline $\mathrm{VC} 1$ & 38,75 & 75,86 & 1,34 & $-0,37$ & $-0,32$ & 0,71 & 0,27 & 0,62 \\
\hline $\mathrm{VC} 2$ & 9,47 & 94,39 & $-0,18$ & 0,03 & 0,50 & $-1,09$ & 0,45 & 0,73 \\
\hline VC3 & 2,35 & 98,99 & 0,16 & $-0,52$ & 1,27 & $-0,56$ & 0,25 & $-0,34$ \\
\hline VC4 & 0,38 & 99,74 & $-0,21$ & 0,23 & $-0,07$ & 1,25 & $-2,00$ & 0,75 \\
\hline VC5 & 0,12 & 99,96 & $-0,04$ & $-0,06$ & $-0,02$ & 0,91 & 0,14 & $-0,01$ \\
\hline VC6 & 0,02 & 100,00 & 0,11 & 1,09 & 0,19 & $-0,25$ & $-0,30$ & $-0,10$ \\
\hline
\end{tabular}

(1)FR: início do florescimento; NRP: número de racemos por planta; CR: comprimento efetivo do racemo primário; AP: altura de plantas; PP: potencial produtivo; TO: teor de óleo das sementes.

Tabela 3. Dissimilaridade entre acessos e cultivares, baseada na distância euclidiana média, obtida a partir dos escores das duas primeiras variáveis canônicas, em mamona.

\begin{tabular}{|c|c|c|c|c|c|c|c|c|}
\hline Acessos e cultivares & 2 & 3 & 4 & 5 & 6 & 7 & 8 & 9 \\
\hline 1. BRA 4871 & 1,65 & 6,57 & 4,44 & 3,99 & 3,42 & 2,58 & 8,28 & 1,25 \\
\hline 2. BRA 2968 & - & 6,74 & 5,30 & 2,36 & 3,59 & 1,08 & 8,97 & 0,42 \\
\hline 3. BRA 5550 & & - & 3,05 & 7,24 & 3,19 & 7,62 & 14,64 & 6,75 \\
\hline 4. BRA 7722 Papo-de-gia & & & - & 6,75 & 2,28 & 6,36 & 11,95 & 5,13 \\
\hline 5. BRS 188 Paraguaçu & & & & - & 4,59 & 1,86 & 10,65 & 2,78 \\
\hline 6. BRS 149 Nordestina & & & & & - & 2,64 & 5,18 & 2,32 \\
\hline 7. IAC-80 & & & & & & - & 8,85 & 1,37 \\
\hline 8. Mirante-10 & & & & & & & - & 8,70 \\
\hline 9. Pernambucana Melhorada & & & & & & & & - \\
\hline
\end{tabular}


Papo-de-gia e BRS 188 Paraguaçu e IAC-80. Cruz et al. (2004) sugerem o não envolvimento de indivíduos de mesmo padrão de dissimilaridade nos cruzamentos, de modo a não restringir a variabilidade genética e assim evitar reflexos negativos nos ganhos a serem obtidos pela seleção. Conforme relatado por Abreu et al. (1999) e Carpentieri-Pípolo et al. (2000), as melhores combinações híbridas, a serem testadas em um programa de melhoramento, devem envolver parentais tanto divergentes como de elevada performance média.

Na Figura 1, encontra-se a dispersão gráfica dos dados referentes aos seis caracteres avaliados nos acessos e cultivares. Os escores foram plotados em espaço bidimensional, com a distância desses pontos

Tabela 4. Análise de agrupamento dos cinco acessos e quatro cultivares, relativa às duas primeiras variáveis canônicas, em mamona.

\begin{tabular}{lllllllll}
\hline Grupo & \multicolumn{7}{c}{ Acessos e cultivares } \\
\hline I & 1 & 2 & 3 & 4 & 5 & 6 & 7 & 9 \\
\hline II & 8 & & & & & & & \\
\hline
\end{tabular}

Acessos: 1: BRA 4781; 2: BRA 2968; 3: BRA 5550; 4: BRA 7722 Papo-de-gia; cultivares: 5: BRS 188 Paraguaçu; 6: BRS 149 Nordestina; 7: IAC-80; 8: Mirante-10; 9: Pernambucana Melhorada.

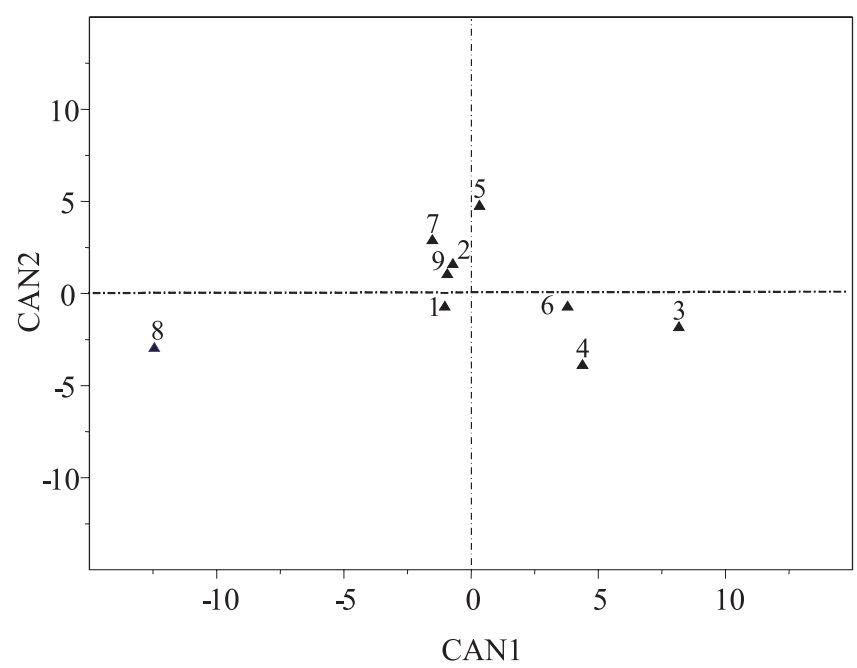

Figura 1. Dispersão gráfica dos escores, em relação aos dois eixos representativos das duas primeiras variáveis canônicas (CAN1 e CAN2), relativas às seis características avaliadas nos quatro acessos e cinco cultivares: 1: BRA 4871; 2: BRA 2968; 3: BRA 5550; 4: BRA 7722 Papo de gia, 5: BRS 188 Paraguaçu; 6: BRS 149 Nordestina; 7: IAC 80; 8: Mirante 10; 9: Pernambucana Melhorada. proporcional ao grau de dissimilaridade entre os genótipos.

A retenção de 94,39\% da variância total dos dados originais, pelas duas primeiras variáveis canônicas, possibilitou a transposição da divergência genética do espaço p-dimensional ( $\mathrm{p}=6$ ) para bidimensional, com grau de distorção desprezível. Resultado semelhante foi obtido por Vidigal et al. (1997) em relação à mandioca.

A distribuição dos acessos e cultivares, no gráfico de dispersão (Figura 1), permitiu identificar como menos divergentes entre si o acesso BRA 2968 e a cultivar Pernambucana Melhorada e, como os mais divergentes, o acesso BRA 5550 e a cultivar Mirante-10. Estes resultados estão de acordo com os obtidos pelo agrupamento de Tocher, o que os indica como confiáveis na identificação de genitores divergentes.

As características de maior importância, na divergência genética entre os acessos e as cultivares foram: início do florescimento (FR), altura da planta (AP), teor de óleo das sementes (TO) e comprimento efetivo do racemo primário (CR), as quais apresentaram maiores coeficientes (autovetores) nas duas primeiras variáveis canônicas.

\section{Conclusões}

1. Existe reduzida variabilidade genética entre os genótipos.

2. A cultivar mais divergente, Mirante-10, não é recomendada para hibridação para formar populações segregantes, em razão de seu baixo desempenho médio, e não são recomendados os acessos e demais cultivares, por apresentarem o mesmo padrão de dissimilaridade.

3. As características de maior importância para a divergência genética são: início do florescimento, altura da planta, teor de óleo das sementes e comprimento efetivo do racemo primário.

4. A característica que contribui menos para a divergência genética é o potencial produtivo, e a característica que nada contribuiu é o número de racemos por planta.

\section{Referências}

ABREU, A. de F.B.; RAMALHO, M.A.P.; FERREIRA D.F. Selection potential for seed yield from intra and inter-racial populations in common bean. Euphytica, v.108, p.121-127, 1999.

CARPENTIERI-PÍPOLO, V.; DESTRO, D.; PRETE, C.E.C.; GONZALES, M.G.N.; POPPER, I.; ZANATTA, S.; SILVA, F.A. 
da. Seleção de genótipos parentais de acerola com base na divergência genética multivariada. Pesquisa Agropecuária Brasileira, v.35, p.1613-1619, 2000

CRUZ, C.D. Aplicações de algumas técnicas multivariadas no melhoramento de plantas. 1990. 188p. Tese (Doutorado) - Escola Superior de Agricultura Luiz de Queiroz, Piracicaba.

CRUZ, C.D.; REGAZZI, A.J.; CARNEIRO, P.C.S. Modelos biométricos aplicados ao melhoramento genético. Viçosa: UFV, 2004. p.223-375.

DIAS, L.A.S.; KAGEYAMA, P.Y. Multivariate genetic divergence and hybrid performance of cacao (Theobroma cacao L.). Revista Brasileira de Genética, v.20, p.63-70, 1997.

FALCONER, D.S. Introdução à genética quantitativa. Viçosa: UFV, 1981. 279p.

FERRÃO, M.A.G.; VIEIRA, C.; CRUZ, C.D.; CARDOSO, A.A. Divergência genética em feijoeiro em condições de inverno tropical. Pesquisa Agropecuária Brasileira, v.37, p.10891098, 2002.

FIGUEREDO NETO, A.; ALMEIDA, F. de A.C.; GOUVEIA, J.P.G. de; NÓBREGA, M.B.M.; CARNEIRO, R.M.; PEDROZA, J.P. Divergência genética em acessos de mamona (Ricinus communis L.) baseada nas características de semente. Revista de Biologia e Ciências da Terra, v.4, p.1-10, 2004.

FREIRE, E.C.; LIMA, E.F.; ANDRADE, F.P. de. Melhoramento genético. In: AZEVÊDO, D.M.P. de; LIMA, E.F. (Ed.). O agronegócio da mamona no Brasil. Brasília: Embrapa Algodão, 2001. p.230-256.

MALUF, W.R.; FERREIRA, P.E.; MIRANDA, J.E.C. Genetic divergence in tomatoes and its relationship with heterosis for yield in $\mathrm{F}_{1}$ hybrids. Revista Brasileira de Genética, v.6, p.453-460, 1983.

MIRANDA, G.V.; COIMBRA, R.R.; GODOY, C.L.; SOUZA, L.V.; GUIMARÃES, L.J.M.; MELO, A.V. de. Potencial de melhoramento e divergência genética de cultivares de milho-pipoca. Pesquisa Agropecuária Brasileira, v.38, p.681-688, 2003.

MIRANDA, J.E.C. de; COSTA, C.P. da; CRUZ, C.D. Predição do comportamento de híbridos de pimentão (Capsicum annum L.) pela divergência genética dos progenitores. Revista Brasileira de Genética, v.11, p.929-937, 1988.
MOREIRA, J.A.N.; LIMA, E.F.; FARIAS, F.J.C.; AZEVÊDO, D.M.P. de. Melhoramento da mamoneira (Ricinus communis L.). Campina Grande: Embrapa-CNPA, 1996. 30p. (Documentos, 44).

NÓBREGA, M.B. de M.; ANDRADE, F.P. de; SANTOS, J.W. dos; LEITE, E.J. Germoplasma. In: AZEVÊDO, D.M.P. de; LIMA, E.F. (Ed.). O agronegócio da mamona no Brasil. Brasília: Embrapa Algodão, 2001. p.257-281.

PARENTE, E.J.S. Biodiesel: uma aventura tecnológica num país engraçado. Fortaleza: Tecbio, 2003. 68p.

PAZ, L.M. Ressonância magnética nuclear (RMN), princípios e aplicações. Óleos e Grãos, v.7, p.32-33, 1996.

RAMALHO, M.A.P.; SANTOS, J.B. dos; ZIMMERMANN, M.J. de O. Genética quantitativa em plantas autógamas: aplicações ao melhoramento do feijoeiro. Goiânia: Ed. da UFG, 1993. p.93-135.

RIBEIRO, F.E.; SOARES, A.R.; RAMALHO, M.A.P. Divergência genética entre populações de coqueiro-gigante-do-Brasil. Pesquisa Agropecuária Brasileira, v.34, p.1615-1622, 1999.

RIBEIRO JÚNIOR, J.I. Análises estatísticas no SAEG. Viçosa: UFV, 2001. 301p.

SAVY FILHO, A. Mamona: tecnologia agrícola. Campinas: Emopi, 2005. 105p.

SAVY FILHO, A. Melhoramento da mamona. In: BORÉM, A. (Ed.). Melhoramento de espécies cultivadas. Viçosa: Universidade Federal de Viçosa, 1999. p.385-407.

SAVY FILHO, A.; BANZATTO, N.V. Mamona. In: FURLANI, A.M.C.; VIÉGAS, G.P. (Ed.). O melhoramento de plantas no Instituto Agronômico. Campinas: Instituto Agronômico, 1993. v.1, p.315-353.

SCOTT, A.J.; KNOTT, M. A cluster analysis method for grouping means in the analysis of variance. Biometrics, v.30, p.507-512, 1974.

VEIGA, R.F.A.; SAVY FILHO, A.; BANZATTO, N.V. Descritores mínimos para caracterização e avaliação de mamoneira (Ricinus communis L.) aplicados no Instituto Agronômico de Campinas. Campinas: Instituto Agronômico, 1989. 16p. (IAC. Boletim técnico, 125).

VIDIGAL, M.C.G.; VIDIGAL FILHO, P.S.; AMARAL JÚNIOR, A.T. do; BRACCINI, A. de L. e. Divergência genética entre cultivares de mandioca por meio de estatística multivariada. Bragantia, v.56, p.263-271, 1997.

Recebido em 26 de outubro de 2005 e aprovado em 28 de agosto de 2006 